\title{
OR-5
}

\section{Evaluation of Antihyperlipidemic Effect of Aqueous and Methanol Extracts of Garcinia Atroviridis in Poloxamer 407-Induced Acute Hyperlipidemic Rats}

\author{
Majed Ahmed Al-Mansoub, Mohd. Zaini Asmawi and Vikneswaran Murugaiyah* \\ Discipline of Pharmacology, School of Pharmaceutical Sciences, Universiti Sains Malaysia, Minden 11800 Penang, \\ Malaysia; E-mail: vicky@usm.my
}

Garcinia atroviridis, a member of Clusiaceae family, has gained much attention in recent years due to its ability to reduce body weight. The present study was carried out to investigate the antihyperlipidemic effects of aqueous and methanol extracts of different parts of G. atroviridis, namely ripe fruit with seeds (A), ripe fruit rind (B), unripe fruit with seeds (C), unripe fruit rind (D), leaf (E), and stem (F) in poloxamer 407-induced acute hyperlipidemic rats. The extracts were suspended in $1 \% \mathrm{CMC}$ and orally administered once a day for 3 days at dose level of $1000 \mathrm{mg} / \mathrm{kg} /$ body weight. In general, the aqueous extract showed better antihyperlipidemic activity than the methanol extracts. The aqueous extract of A was the most active extract, which showed significant reduction in serum total cholesterol of $71.59 \%(P<0.05)$ and triglycerides of $93.77 \%(P<0.01)$ compared to hyperlipidemic control. The antihyperlipidemic activity of aqueous extract of A was comparable to that of atorvastatin (60 $\mathrm{mg} / \mathrm{kg}$ ). Further study on the antihyperlipidemic effect of the aqueous extract of A in high fat diet-induced chronic hyperlipidemic rats is ongoing.

Keywords: Garcinia atroviridis, antihyperlipidemic, total cholesterol, triglycerides. 